\title{
Determination of Phase-Eigenvalues by Rational Factorization and Enhanced Simulation of Two-Phase Mass Flow
}

\author{
Puskar R. Pokhrel ${ }^{1,2}$, Bhadra Man Tuladhar ${ }^{1}$ \\ ${ }^{1}$ Department of Mathematics, School of Science, Kathmandu University, Dhulikhel, Kavre, Nepal \\ 2 Department of Mathematics, RR Campus, Tribhuvan University, Kathmandu, Nepal \\ Correspondence to: prpokharel@student.ku.edu.np/prpokharel@ratnarajyalaxmicampus.edu.np
}

\begin{abstract}
In this paper, we present simple and exact eigenvalues for both the solid-and fluid-phases of the real two-phase general model developed by Pudasaini (2012); we call these phase-eigenvalues, the solidphase-eigenvalues and the fluid-phase-eigenvalues. Results are compared by applying the derived phaseeigenvalues that incorporate the phase-interactions in the two-phase debris movements against the simple and classical solid and fluid eigenvalues without any phase interaction. We have constructed several different set of eigenvalues including the coupled phase eigenvalues by using rational factorization method. At first, we consider for general debris height; factorizing the solid and fluid lateral pressure contributions by considering the negligible pressure gradient; negligible solid lateral pressure; negligible fluid lateral pressure; negligible solid and fluid lateral pressure. Secondly, for a thin debris flow height, we also construct the fourth set of eigenvalues in three different cases. These phase-eigenvalues incorporate strong interaction between the solid and fluid dynamics. The simulation results are produced by taking all these different sets of coupled phase-eigenvalues and are compared with the classical uncoupled set of solid and fluid eigenvalues. The results indicate the importance of phase-eigenvalues and supports for a complete description of the phaseeigenvalues for the enhanced description of real two-phase debris flows and landslide motions.
\end{abstract}

Keywords: Debris flows, Exact eigenvalues, Phase-eigenvalues, Rational factorization, Phase-interaction, Simulation

\section{Introduction}

A debris flow is a moving mass that generally consists of loose sand, soil, rock, and water and travels down a slope mainly under the influence of gravity $[12,13,14,16,17,28,30,36,40,43,44$. Debris flows which generally occur in mountainous areas throughout the world are extremely destructive and dangerous natural events. A debris flow can also be caused by a sudden surge of water, caused by the breaking off of a dam, rock fall or a landslide into a lake, ocean or river causing the water to overflow [5, 6, 10, 24, 25, 26, 37, 38, 46, As the flood surge rushes down a dry channel, it can transform into a debris flow by eroding the bed and picking up material on its way [11, 15, 22, 41]. During the event, the material undergoes rapid motions and large deformations, which may cause the boundary to change abruptly or continuously. Some debris flows are very fast - these are the ones that attract more attention. In areas of very steep slopes they can reach speeds of over $160 \mathrm{~km} /$ hour [21].

Every year throughout the world, debris flows cause an immense amount of damage to people and their properties [39]. The growth of population and development activities inevitably puts a lot of pressure on developers to build houses and civil infrastructures in areas that are exposed to elevated risk of snow avalanches, debris flows, mud flows, landslides and similar events which are often of a hydro-geological nature [36]. Debris flow catch dams, flexible wire barriers and land planning measures (hazard maps) are some ways for protecting a region from the destructive debris flow [36. So, the mechanics of debris flows has been a subject of intensive research, the primary goal being to understand the factors leading to debris initiation and finding an appropriate physical and dynamical description of debris flow motion. From a modelling point of view, debris flows are characterized as gravity-driven flows of a multi phase material[12, 13, 14, 16, 17, 20, 28, 30, 35, 36, 40, 43, 44.

In the past, there were various rheological models presented to study the debris flows, e.g., single phase, mixture flows, two layered flows and two-fluid flows. As the two-phase debris flows are advective-diffusive processes, these events are modelled by some complex hyperbolic-parabolic partial differential equations. Pudasaini (2012) proposed an advanced and comprehensive general two-phase debris flow model that includes several dominant physical aspects of the flow including buoyancy, solid-volume fraction-gradient- 
enhanced non-Newtonian viscous stress, virtual mass, and generalized drag. There are strong couplings between the solid and the fluid momentum transfer. Due to its general and comprehensive nature, here, the analysis is based on this general model. We consider the mass and momentum balance equations for the solid- and the fluid-phases. The equations are then written in a matrix form with the vector of the virtual mass induced generalized conservative variables, vector of transport fluxes and the source vector. Recently, Pokhrel et al. (2018) have developed a generalized quasi two-phase mixture model for mass flow which captures all dynamics of the generalized two phase-mass flow model 35. Based on the generalized mixture mass flow model, stream function, vorticity transport, and pressure Poisson equations have been derived [33. The new derived model equations also capture all physical phenomena of the mixture model equation [32].

Landslides, debris flows and tsunamis are large gravitational mass movements and extremely destructive natural hazards that often occur as the motion of the mixture of soil, rock and fluid down mountain slopes 20]. So, reliable methods are needed to accurately predict the flow evolution, run-out distances, inundation areas, deposition behaviour, impact forces, and the overall flow dynamics from inception to standstill; this is required for the prevention measures and enhanced mitigation strategies in geo-hazard-prone areas. Depending on the material involved and the flow dynamics and the momentum transfer between the constituents, these flows can be effectively single phase, or must be described by applying the real two-phase mass flow models; for the correct and reliable description of flow behaviour, we need the exact description of eigenvalues of the system. The correct and reliable description of flow behaviour requires the exact description of eigenvalues of the system [35]. Here, we mainly focus on the derivation, discussion and simulations of some simple analytical solid- and fluid-phase eigenvalues. Phase-eigenvalues are obtained from the flux-matrix formed by taking the rate of change of vector of transport fluxes with respect to the vector of conservative state variables.

Some eigenvalues were discussed on the eigen-structure of two-phase and multi-layer flows can be found in, including, Audusse (2005), Pitman and Le (2005), Bouchut and Morales (2008), Fernandez-Nieto et al. (2008), Pelanti et al.(2008), Castro et al. (2010), and Castro-Diaz et al. (2011). The eigenvalues of the two-phase model were analyzed in Pudasaini (2012) by assuming solid-phase velocity is much greater than the fluid-phase velocity. We have also obtained one set of the eigenvalue by using much less assumption than in Pudasaini (2012). From our eigenvalues, we have also obtained the usual eigenvalues of single-phase granular flow by neglecting the fluid flows debris height, which resembles with the solid-phase eigenvalues as obtained in Pudasaini and Hutter (2007).

From eigenvalues, we can extract wave speeds and determine the Froude number ( $\mathrm{Fr}$ ). As the wave speeds for both the solid and fluid phases carry very important information of the flow dynamics and thus play crucial role in the simulation, their accurate knowledge plays vital role in correctly describing real twophase flows. Our simulation results show that all of our coupled phase-eigenvalues provide enhanced flow dynamics as compared to the classical uncoupled solid- only and fluid- only eigenvalues.

\section{Model Equations}

The two-phase debris flows are characterized by its distinct properties of the materials involved (Pudasaini, 2012): the fluid phase is characterized by its density $\rho_{f}$, viscosity $\eta_{f}$, and isotropic stress distribution; the solid phase is characterized by its density $\rho_{s}$, internal and basal friction angles $\phi$ and $\delta$, respectively, and anisotropic stress distribution, $K$ (lateral earth pressure coefficient). These characterizations and the presence of relative motion between the two phases lead to different mass and momentum balance equations for the solid and the fluid phases, respectively. Let $u_{s}, u_{f}$ and $\alpha_{s}, \alpha_{f}\left(=1-\alpha_{s}\right)$ denote the slope parallel velocities, and volume fractions for the solid and the fluid constituents, denoted by the suffices $s$ and $f$, respectively. The general two-phase debris flow model when reduced to one-dimensional channel flows consists of the depth-averaged mass and momentum balance equations for the solid and fluid phases, as 
described by the following set of partial differential equations [35]:

$$
\begin{aligned}
& \frac{\partial}{\partial t}\left(\alpha_{s} h\right)+\frac{\partial}{\partial x}\left(\alpha_{s} h u_{s}\right)=0, \quad \frac{\partial}{\partial t}\left(\alpha_{f} h\right)+\frac{\partial}{\partial x}\left(\alpha_{f} h u_{f}\right)=0, \\
& \frac{\partial}{\partial t}\left[\alpha_{s} h\left(u_{s}-\gamma \mathcal{C}\left(u_{f}-u_{s}\right)\right)\right]+\frac{\partial}{\partial x}\left[\alpha_{s} h\left(u_{s}^{2}-\gamma \mathcal{C}\left(u_{f}^{2}-u_{s}^{2}\right)+\frac{\beta_{s} h}{2}\right)\right]=h S_{s}, \\
& \frac{\partial}{\partial t}\left[\alpha_{f} h\left(u_{f}+\frac{\alpha_{s}}{\alpha_{f}} \mathcal{C}\left(u_{f}-u_{s}\right)\right)\right]+\frac{\partial}{\partial x}\left[\alpha_{f} h\left(u_{f}^{2}+\frac{\alpha_{s}}{\alpha_{f}} \mathcal{C}\left(u_{f}^{2}-u_{s}^{2}\right)+\frac{\beta_{f} h}{2}\right)\right]=h S_{f} .
\end{aligned}
$$

The source term in the $x$-momentum equation for solid is

$$
S_{s}=\alpha_{s}\left[g^{x}-\frac{u_{s}}{\left|\mathbf{u}_{\mathbf{s}}\right|} \tan \delta p_{b_{s}}-\epsilon p_{b_{s}} \frac{\partial b}{\partial x}\right]-\epsilon \alpha_{s} \gamma p_{b_{f}}\left[\frac{\partial h}{\partial x}+\frac{\partial b}{\partial x}\right]+C_{D G}\left(u_{f}-u_{s}\right)\left|\mathbf{u}_{f}-\mathbf{u}_{s}\right|^{J-1} .
$$

The source term in the $x$-momentum equation for fluid is

$$
\begin{aligned}
S_{f}= & \alpha_{f}\left[g^{x}-\epsilon\left[\frac{1}{h} \frac{\partial}{\partial x}\left(\frac{h^{2}}{2} p_{b_{f}}\right)+p_{b_{f}} \frac{\partial b}{\partial x}-\frac{1}{\alpha_{f} N_{R}}\left\{2 \frac{\partial^{2} u_{f}}{\partial x^{2}}-\frac{\chi u_{f}}{\epsilon^{2} h^{2}}\right\}\right.\right. \\
& \left.\left.+\frac{1}{\alpha_{f} N_{R_{\mathcal{A}}}}\left\{2 \frac{\partial}{\partial x}\left(\frac{\partial \alpha_{s}}{\partial x}\left(u_{f}-u_{s}\right)\right)\right\}-\frac{\xi \alpha_{s}\left(u_{f}-u_{s}\right)}{\epsilon^{2} \alpha_{f} N_{R_{\mathcal{A}}} h^{2}}\right]\right]-\frac{1}{\gamma} C_{D G}\left(u_{f}-u_{s}\right)\left|\mathbf{u}_{f}-\mathbf{u}_{s}\right|^{J-1} .
\end{aligned}
$$

where $J=1$ (linear drag) or 2 (quadratic drag) and the other parameters are

$$
\begin{aligned}
& \beta_{s}=\epsilon K p_{b_{s}}, \quad \beta_{f}=\epsilon p_{b_{s}}, \quad p_{b_{f}}=-g^{z}, \quad p_{b_{s}}=(1-\gamma) p_{b_{f}}, \quad C_{D G}=\frac{\alpha_{s} \alpha_{f}(1-\gamma)}{\left[\epsilon \mathcal{U}_{T}\left\{\mathcal{P} \mathcal{F}\left(R e_{p}\right)+(1-\mathcal{P}) \mathcal{G}\left(R e_{p}\right)\right\}\right]}, \\
& \mathcal{F}=\frac{\gamma}{180}\left(\frac{\alpha_{f}}{\alpha_{s}}\right)^{3} R e_{p}, \mathcal{G}=\alpha_{f}^{M\left(R e_{p}\right)-1}, \quad \gamma=\frac{\rho_{f}}{\rho_{s}}, R e_{p}=\frac{\rho_{f} d \mathcal{U}_{T}}{\eta_{f}}, \quad N_{R}=\frac{\sqrt{g L} H \rho_{f}}{\alpha_{f} \eta_{f}}, \\
& N_{R_{\mathcal{A}}}=\frac{\sqrt{g L} H \rho_{f}}{\mathcal{A} \eta_{f}}, \quad \alpha_{f}=1-\alpha_{s}, \quad \mathcal{A}=\mathcal{A}\left(1-\alpha_{s}\right) .
\end{aligned}
$$

Here, $h$ is the flow depth, are associated with the effective solid and fluid pressure at the base, $x$ and $z$ are coordinates along the flow directions, and $g^{x}$ and $g^{z}$ are the components of gravitational acceleration along the $x$ and $z$ directions respectively, and $\beta_{s}$ and $\beta_{f}$ are the lateral hydraulic pressure parameters for solid and fluid respectively. $L$ and $H$ are the typical length and depth of the flow, $\epsilon=H / L$ is the aspect ratio, and $\mu=\tan \delta$ is the basal friction coefficient. $C_{D G}$ is the generalized drag coefficient, $\mathcal{U}_{T}$ is the terminal velocity of a particle and $P \in[0,1]$ is a parameter which combines the solid-like $(\mathcal{G})$ and fluid-like $(\mathcal{F})$ drag contributions to flow resistance, $\gamma$ is the density ratio, $\mathcal{C}$ is the virtual mass coefficient (solid particles induced kinetic energy of fluid phase), $\eta_{f}$ is the fluid viscosity and $M$ is a function of the particle Reynolds number $\left(R_{e_{p}}\right)$, $\chi$ includes vertical shearing of fluid velocity, $\xi$ takes into account of different distributions of $\alpha_{s}, A=A\left(\alpha_{f}\right)$ is the mobility of the fluid at the interface [35], and $N_{R}$ and $N_{R_{A}}$ are Reynolds numbers associated with the classical Newtonian, and enhanced non-Newtonian fluid viscous stresses respectively [37, 38. Also, slope topography is represented by $b=b(x)$.

There are two important aspects of the model equations (1)-(3). Firstly, the inertial terms on the left hand side of (2)-(3) include the lateral pressure (associated with $s$ and $f$ ) and the virtual mass coefficient $C$. Secondly, the source in the solid momentum has three different contributions: (i) gravity, the Coulomb friction and the slope gradient (first square brackets), (ii) terms associated with the buoyancy force (second square brackets), and (iii) the generalized drag contribution $\left(C_{D G}\right)$ (last term).

The source term for the fluid momentum equation (4) has six different contributions: The first three terms emerge from the gravity load (first term), the fluid pressure gradient at the bed (second term), and the fluid pressure applied to the topographic gradient (third term), the fourth and fifth group of the terms associated with $N_{R}$ and $N_{R_{A}}$ are the Newtonian viscous, and the solid-volume-fraction-gradient-enhanced 
non-Newtonian viscous stresses respectively, the last term is due to the drag force. The term associated with $\beta_{s}$ in (2) accounts for the buoyancy-reduced lateral pressure. The solid load is reduced by the buoyancy force by the factor $(1-\gamma)$ as seen in $p_{b_{s}}$, Coulomb friction and in the drag term, $C_{D G}$. The non-dimensional number $N_{R_{A}}$ is termed as the mobility number [35].

\section{Characteristic Speeds and Critical Flows}

The characteristic speeds are determined from the eigenvalues of the system. In the model equations (1)-(3), the solid and fluid phase velocities $u_{s}, u_{f}$, and the solid and fluid volume fractions, $\alpha_{s}, \alpha_{f}$, can be expressed as $u_{s}=m_{s} / h_{s}, u_{f}=m_{f} / h_{f}, \alpha_{s}=h_{s} / h, \alpha_{f}=h_{f} / h$, so that $\alpha_{s} / \alpha_{f}=h_{s} / h_{f}$, where $m_{s}=h \alpha_{s} u_{s}$ and $m_{f}=h \alpha_{f} u_{f}$ are solid and fluid component momentum fluxes respectively. With these relations, equations (1)- (3) can be put in the matrix form as

$$
\frac{\partial \mathbf{T}(\mathbf{W})}{\partial t}+\frac{\partial \mathbf{F}(\mathbf{W})}{\partial x}=\mathbf{S}(\mathbf{W})
$$

where $\mathbf{T}=\mathbf{T}(\mathbf{W})$ is the vector of the virtual mass induced generalized conservative variables, $\mathbf{F}=\mathbf{F}(\mathbf{W})$ is the vector of transport fluxes in the $x$-direction with:

$$
\mathbf{T}=\left[\begin{array}{c}
h_{s} \\
h_{f} \\
m_{s}-\gamma \mathcal{C}\left(\frac{m_{f} h_{s}}{h_{f}}-m_{s}\right) \\
m_{f}+\mathcal{C}\left(\frac{m_{f} h_{s}}{h_{f}}-m_{s}\right)
\end{array}\right], \mathbf{F}=\left[\begin{array}{c}
m_{s} \\
m_{f} \\
\frac{m_{s}^{2}}{h_{s}}+\frac{1}{2} \beta_{s} h_{s}\left(h_{s}+h_{f}\right)-\gamma \mathcal{C}\left(\frac{m_{f}^{2} h_{s}}{h_{f}^{2}}-\frac{m_{s}^{2}}{h_{s}}\right) \\
\frac{m_{f}^{2}}{h_{f}}+\frac{1}{2} \beta_{f} h_{f}\left(h_{f}+h_{s}\right)+\mathcal{C}\left(\frac{m_{f}^{2} h_{s}}{h_{f}^{2}}-\frac{m_{s}^{2}}{h_{s}}\right)
\end{array}\right], \mathbf{W}=\left[\begin{array}{c}
h_{s} \\
h_{f} \\
m_{s} \\
m_{f}
\end{array}\right]
$$

For simplicity, and only for the purpose of generating simple but exact eigenvalues, the virtual mass coefficient $\mathcal{C}$ is set to zero. Then, $\mathbf{T} \rightarrow \mathbf{W}$, and $\mathbf{F}$ takes the simple form:

$$
\mathbf{F}=\left[\begin{array}{c}
m_{s} \\
m_{f} \\
\frac{m_{s}^{2}}{h_{s}}+\frac{1}{2} \beta_{s} h_{s}\left(h_{s}+h_{f}\right) \\
\frac{m_{f}^{2}}{h_{f}}+\frac{1}{2} \beta_{f} h_{f}\left(h_{f}+h_{s}\right)
\end{array}\right], \quad \mathbf{W}=\left[\begin{array}{c}
h_{s} \\
h_{f} \\
m_{s} \\
m_{f}
\end{array}\right]
$$

The mass and the momentum fluxes for the solid and the fluid are $m_{s}, m_{f}$; and the depth-averaged momentum fluxes $\frac{m_{s}^{2}}{h_{s}}+\frac{1}{2} \beta_{s} h_{s}\left(h_{s}+h_{f}\right), \frac{m_{f}^{2}}{h_{f}}+\frac{1}{2} \beta_{f} h_{f}\left(h_{f}+h_{s}\right)$ for both components. One of the main tasks here is to obtain the eigenvalues of the system (6) as characterized by (8). The eigenvalues are associated with the matrix

$$
\mathbf{A}=\frac{\partial \mathbf{F}}{\partial \mathbf{W}}=\left[\begin{array}{cccc}
0 & 0 & 1 & 0 \\
0 & 0 & 0 & 1 \\
-u_{s}^{2}+\beta_{s} \alpha_{s} h+\frac{1}{2} \beta_{s} \alpha_{f} h & \frac{1}{2} \beta_{s} \alpha_{s} h & 2 u_{s} & 0 \\
\frac{1}{2} \beta_{f} \alpha_{f} h & -u_{f}^{2}+\beta_{f} \alpha_{f} h+\frac{1}{2} \beta_{f} \alpha_{s} h & 0 & 2 u_{f}
\end{array}\right]
$$

The eigenvalues of $\mathbf{A}$ are given by the roots of the characteristics polynomial equation $|\mathbf{A}-\lambda \mathbf{I}|=0$ in $\lambda$; the roots for $\lambda$ are given by

$$
\left(-u_{s}^{2}+\beta_{s} \alpha_{s} h+\frac{1}{2} \beta_{s} \alpha_{f} h+2 \lambda u_{s}-\lambda^{2}\right)\left(-u_{f}^{2}+\beta_{f} \alpha_{f} h+\frac{1}{2} \beta_{f} \alpha_{s} h+2 \lambda u_{f}-\lambda^{2}\right)=\frac{1}{4} \beta_{s} \beta_{f} \alpha_{s} \alpha_{f} h^{2} .
$$




\section{Determination of Eigenvalues By Rational Factorization}

The last term of (9) generates from the lateral hydraulic pressure gradients for the solid $\left(0.5 \beta_{s} \alpha_{s} h\right)$ and for the fluid $\left(0.5 \beta_{f} \alpha_{f} h\right)$; these terms may play dominant role in determining the eigenvalues of the system during the flow inception, flow obstacle interactions, diverging and converging flows, and during the deposition process; the terms $\beta_{s}$ and $\beta_{f}$ are the lateral hydraulic pressure parameters for solid and fluid respectively, whereas $\alpha_{s}$ and $\alpha_{f}$ are the solid and fluid volume fractions which are less than unity. But the depth of debris may be less than or equal to, or greater than unity; so, we may consider two special situations, namely $h>0$ and $0<h<1$.

\subsection{For general debris height; i.e., $\mathrm{h}>0$}

The total debris height is always greater than zero. Here, in order to obtain some easily computable /obtainable eigenvalues, we propose two "rational factorizations". The essence of these rational factorizations can be justified later after getting the more complicated general eigenvalues for the solid and the fluid phases. Based on a generalized two-phase mass flow model [35], we are motivated to apply rational factorization that paves the way for obtaining quick and technical eigenvalues against the much more complicated eigenvalues. This provides us with some basic and fundamental understanding of the much more complex system. But, this procedure do not fully follow the mathematical rigorousness.

\section{Factorizing into solid and fluid lateral pressure contributions}

Based on the physical process involved in the two-phase solid and the fluid mixture, by inspection, the term on the right hand side in $(9)$ can be factorized into the solid and fluid lateral pressure contributions as

$$
\frac{1}{4} \beta_{s} \beta_{f} \alpha_{s} \alpha_{f} h^{2}=\left(\frac{1}{2} \beta_{s}\left(h \alpha_{s}\right)\right)\left(\frac{1}{2} \beta_{f}\left(h \alpha_{f}\right)\right)=\left( \pm \frac{1}{2} \beta_{s} h_{s}\right)\left( \pm \frac{1}{2} \beta_{f} h_{f}\right) .
$$

Here, for consistency, the sign must be taken either both positive, or both negative. Then, (9) can be written as

$$
\left(-u_{s}^{2}+\beta_{s}\left(\alpha_{s} h\right)+\frac{1}{2} \beta_{s}\left(\alpha_{f} h\right)+2 \lambda u_{s}-\lambda^{2}\right)\left(-u_{f}^{2}+\beta_{f} \alpha_{f} h+\frac{1}{2} \beta_{f} \alpha_{s} h+2 \lambda u_{f}-\lambda^{2}\right)=\left( \pm \frac{1}{2} \beta_{s} h_{s}\right)\left( \pm \frac{1}{2} \beta_{f} h_{f}\right)
$$

Following the structure and involved variables, our experience tell that the expression in the first bracket on the left hand side of $(9)$ is mainly associated with the solid constituent. Similarly, the second bracket is associated with the fluid constituent. Therefore, motivated from this, and from a practical point of view, (9) can effectively be factorized into solid and fluid contributions, with the associated eigenvalues for solid $\left(\lambda_{s}\right)$ and the eigenvalues for the fluid $\left(\lambda_{f}\right)$, as:

$$
\begin{aligned}
& \left(-u_{s}^{2}+\beta_{s}\left(\alpha_{s} h\right)+\frac{1}{2} \beta_{s}\left(\alpha_{f} h\right)+2 \lambda_{s} u_{s}-\lambda_{s}^{2}\right)=\left( \pm \frac{1}{2} \beta_{s} h_{s}\right) \\
& \left(-u_{f}^{2}+\beta_{f}\left(\alpha_{f} h\right)+\frac{1}{2} \beta_{f}\left(\alpha_{s} h\right)+2 u_{f} \lambda_{f}-\lambda_{f}^{2}\right)=\left( \pm \frac{1}{2} \beta_{f} h_{f}\right) .
\end{aligned}
$$

These equations can be written as quadratic equations in $\lambda_{s}$ and $\lambda_{f}$ as

$$
\begin{aligned}
& \lambda_{s}^{2}-2 \lambda_{s} u_{s}+\left(u_{s}^{2}-\beta_{s} h_{s}-\frac{1}{2} \beta_{s} h_{f} \pm \frac{1}{2} \beta_{s} h_{s}\right)=0 \\
& \lambda_{f}^{2}-2 u_{f} \lambda_{f}+\left(u_{f}^{2}-\beta_{f} h_{f}-\frac{1}{2} \beta_{f} h_{s} \pm \frac{1}{2} \beta_{f} h_{f}\right)=0 .
\end{aligned}
$$


Solving the equation 10 for $\lambda_{s}$, and 11 for $\lambda_{f}$, we get

$$
\begin{aligned}
& \lambda_{s}=u_{s} \pm \sqrt{u_{s}^{2}-\left(u_{s}^{2}-\beta_{s} h_{s}-\frac{1}{2} \beta_{s} h_{f} \pm \frac{1}{2} \beta_{s} h_{s}\right)}, \\
& \lambda_{f}=u_{f} \pm \sqrt{u_{f}^{2}-\left(u_{f}^{2}-\beta_{f} h_{f}-\frac{1}{2} \beta_{f} h_{f} \pm \frac{1}{2} \beta_{f} h_{f}\right)} .
\end{aligned}
$$

The first set of eigenvalues: Taking the positive sign inside the radicals in 12 and $(13)$, the first set of eigenvalues for solid and fluid phases, respectively, are

$$
\lambda_{s}=u_{s} \pm \sqrt{0.5 \beta_{s}\left(h_{s}+h_{f}\right)}, \quad \lambda_{f}=u_{f} \pm \sqrt{0.5 \beta_{f}\left(h_{s}+h_{f}\right)} .
$$

Single-phase classical eigenvalues: Equations $(14)$ are more general eigenvalues as they reduce to the classical eigenvalues for solid-only (flows of granular materials), and fluid-only (flows of fluid) when $h_{s} \approx h_{f}$ [36, ( see, 15) )below. If $h_{s} \approx h_{f}$, they imply usual eigenvalues for single-phase (solid) and single-phase (fluid), separately, as:

$$
\lambda_{s}=u_{s} \pm \sqrt{\beta_{s} h_{s}}, \quad \lambda_{f}=u_{f} \pm \sqrt{\beta_{f} h_{f}}
$$

The second set of eigenvalues: Taking the negative sign inside the radicals in $(12)$ and $(13)$, the second set of eigenvalues for solid and fluid phases, respectively, are

$$
\lambda_{s}=u_{s} \pm \sqrt{0.5 \beta_{s}\left(h_{s}+h_{f}\right)+\beta_{s} h_{s}}, \quad \lambda_{f}=u_{f} \pm \sqrt{0.5 \beta_{f}\left(h_{s}+h_{f}\right)+\beta_{f} h_{f}} .
$$

Comparison between the first and second sets of eigenvalues: It is interesting to compare the solidand fluid-phase wave speeds (expressions with radicals) in (14) and (16). Wave speeds in (14) are reduced by $\beta_{s} h_{s}, \beta_{f} h_{f}$, as compared to the same in $(16)$. In $(16)$, the respective wave speeds are just the one half of the speeds of the debris bulk, because $h_{s}+h_{f}=h$. However, in 15 the respective solid and fluid wave speeds are the classical wave speeds, namely, $\beta_{s} h_{s}$ for solid, and $\beta_{f} h_{f}$ for fluid, that are enhanced by the one half of wave speeds of the debris bulk with respect to $\beta_{s}$ and $\beta_{f}$.

\section{Assigning equal factors}

Another possibility to factorize the term on the right hand side of $(9)$ is simply to factorize the whole term as:

$$
\frac{1}{4} \beta_{s} \beta_{f} \alpha_{s} \alpha_{f} h^{2}=\left(\frac{1}{2} \beta_{s}\left(h \alpha_{s}\right)\right)\left(\frac{1}{2} \beta_{f}\left(h \alpha_{f}\right)\right)=\left( \pm \frac{1}{2} \sqrt{\beta_{s} h_{s} \beta_{f} h_{f}}\right)^{2} .
$$

So, the equation 9 can be written as

$$
\left(-u_{s}^{2}+\beta_{s}\left(\alpha_{s} h\right)+\frac{1}{2} \beta_{s}\left(\alpha_{f} h\right)+2 \lambda u_{s}-\lambda^{2}\right)\left(-u_{f}^{2}+\beta_{f} \alpha_{f} h+\frac{1}{2} \beta_{f} \alpha_{s} h+2 \lambda u_{f}-\lambda^{2}\right)=\left( \pm \frac{1}{2} \sqrt{\beta_{s} h_{s} \beta_{f} h_{f}}\right)^{2} .
$$

It is natural to assume that the lateral solid and fluid pressures $\left(\beta_{s} h_{s}\right)$ and $\left(\beta_{f} h_{f}\right)$, influence the dynamics of one another. Then, it is legitimate to factorize the above expression into the solid and fluid structures, as

$$
\begin{array}{r}
\left(-u_{s}^{2}+\beta_{s}\left(\alpha_{s} h\right)+\frac{1}{2} \beta_{s}\left(\alpha_{f} h\right)+2 \lambda_{s} u_{s}-\lambda_{s}^{2}\right)= \pm \frac{1}{2} \sqrt{\beta_{s} h_{s} \beta_{f} h_{f}}, \\
\left(-u_{f}^{2}+\beta_{f}\left(\alpha_{f} h\right)+\frac{1}{2} \beta_{f}\left(\alpha_{s} h\right)+2 u_{f} \lambda_{f}-\lambda_{f}^{2}\right)= \pm \frac{1}{2} \sqrt{\beta_{s} h_{s} \beta_{f} h_{f}}, \\
\lambda_{s}^{2}-2 \lambda_{s} u_{s}+\left(u_{s}^{2}-\beta_{s} h_{s}-\frac{1}{2} \beta_{s} h_{f}\right) \pm \frac{1}{2} \sqrt{\beta_{s} \beta_{f} h_{s} h_{f}}=0, \\
\lambda_{f}^{2}-2 u_{f} \lambda_{f}+\left(u_{f}^{2}-\beta_{f} h_{f}-\frac{1}{2} \beta_{f} h_{s}\right) \pm \frac{1}{2} \sqrt{\beta_{s} \beta_{f} h_{s} h_{f}}=0 .
\end{array}
$$


The third set of eigenvalues: Solving (17) for $\lambda_{s}$ and (18) $\lambda_{f}$, the third set of eigenvalues for the solid and fluid phases, respectively, are

$$
\lambda_{s}=u_{s} \pm \sqrt{\beta_{s}\left(h_{s}+0.5 h_{f}\right) \mp \frac{1}{2} \sqrt{\beta_{s} \beta_{f} h_{s} h_{f}}}, \quad \lambda_{f}=u_{f} \pm \sqrt{\beta_{f}\left(h_{f}+0.5 h_{s}\right) \mp \frac{1}{2} \sqrt{\beta_{s} \beta_{f} h_{s} h_{f}}} .
$$

These are more general eigenvalues of the system (6) than those in (14) and (16).

Simplified eigenvalues: From the eigenvalues in (19), we can obtain some nice and simplified eigenvalues by assuming $h_{s} \approx h_{f}$, and $\beta_{s} \approx \beta_{f}$, then, the solid and the fluid eigenvalues, respectively, reduce to:

$$
\lambda_{s}=u_{s} \pm \sqrt{\beta_{s} h_{s}+0.5 \beta_{s}\left(h_{s} \pm h_{s}\right)}, \quad \lambda_{f}=u_{f} \pm \sqrt{\beta_{f} h_{f}+0.5 \beta_{f}\left(h_{f} \pm h_{f}\right)} .
$$

Hence, the reduced and simple eigenvalues (first set for minus sign and second set for plus sign) are:

$$
\begin{array}{ll}
\lambda_{s}=u_{s} \pm \sqrt{\beta_{s} h_{s}} ; & \lambda_{s}=u_{s} \pm \sqrt{2 \beta_{s} h_{s}} \\
\lambda_{f}=u_{f} \pm \sqrt{\beta_{f} h_{f}} ; & \lambda_{f}=u_{f} \pm \sqrt{2 \beta_{f} h_{f}} .
\end{array}
$$

This implies that (16), (19) and 200(right column) are the enhanced eigenvalues whereas (13) and 200) (left column) are somehow reduced eigenvalues.

\subsubsection{Negligible pressure gradient}

In simple situations, at least one of the lateral hydraulic pressure gradients may be negligible, which can lead to three different situations, each justifies the neglection of the last term in (9); this paves the way for the direct and immediate factorization of 99 .

\section{A. Negligible solid lateral pressure $\beta_{s} h_{s} / 2 \sim 0$ :}

This may be an observable situation even in natural two-phase flows when the wave or the surge of a debris flow propagates in which the distribution of solid can be approximated to be uniform along the downslope direction. However, due to the surge (primary, or secondary), and thus the changing geometric profile of the debris, the lateral hydraulic pressure for the fluid may not be negligible. Alternatively, if some part of the debris flow is locally dominated by the fluid component, e.g., say the tail, then $\alpha_{s} \sim 0$. This, then minimizes the effect of the solid pressure gradient to zero.

\section{B. Negligible fluid lateral pressure, $\beta_{f} h_{f} / 2 \sim 0$ :}

This is similar to A, but in a contrasting situation when the solid is replaced by the fluid. In particular, if locally some part of the debris is solid dominated, say, e.g., the leading head, then $\alpha_{f} \sim 0$. This, then minimizes the effect of the fluid pressure gradient to zero.

C. Negligible solid and fluid lateral pressure, $\left(\beta_{s} h_{s} / 2\right)\left(\beta_{f} h_{f} / 2\right) \ll 1$ :

This may happen in some particular situations when both the solid and the fluid lateral pressure gradients are small (say less than unity); this is the case, specially in more flat flows; then, their product can be neglected.

\subsection{For a thin debris flow height, i.e., when $0<h<1$}

The height of the debris flow can be assumed relatively thin, e.g., for small volume. This may be a valid assumption after the flow travels a sufficient down slope distance and before it comes to the sudden deposition. This means that this approximation can be more accurate in the long and main flow path. We also note that, here the flow variables and parameters are non-dimensional. The solid and fluid volume fractions $\alpha_{s}, \alpha_{f}$ are less than unity and the earth pressure coefficients $\beta_{s}$ and $\beta_{f}$ are also less than unity, 
then the last term, i.e., $\frac{1}{4} \alpha_{s} \alpha_{f} \beta_{s} \beta_{f} h^{2}$ in $[9]$ can be neglected. With this, the complexity is now reduced heavily. So, 9 can be written as

$$
\left(-u_{s}^{2}+\beta_{s} \alpha_{s} h+\frac{1}{2} \beta_{s} \alpha_{f} h+2 \lambda u_{s}-\lambda^{2}\right)\left(-u_{f}^{2}+\beta_{f} \alpha_{f} h+\frac{1}{2} \beta_{f} \alpha_{s} h+2 \lambda u_{f}-\lambda^{2}\right)=0 .
$$

This is a prefect factorization which facilitates direct solution for the solid and the fluid eigenvalues. As before, based on their dynamics, we assign the solid and fluid eigenvalue variables $\lambda_{s}$ and $\lambda_{f}$ to the first and second brackets on the left hand side of (21). So, we get quadratic equations in $\lambda_{s}$ and $\lambda_{f}$ as follows:

$$
\begin{aligned}
& \lambda_{s}^{2}-2 \lambda_{s} u_{s}+\left(u_{s}^{2}-\beta_{s} h_{s}-0.5 \beta_{s} h_{f}\right)=0 \\
& \lambda_{f}^{2}-2 \lambda u_{f}+\left(u_{f}^{2}-\beta_{f} h_{f}-0.5 \beta_{f} h_{s}\right)=0 .
\end{aligned}
$$

Solving (22) for $\lambda_{s}$ and 23 for $\lambda_{f}$, the third set of eigenvalues for the solid and fluid phases, respectively, are

$$
\lambda_{s}=u_{s} \pm \sqrt{\beta_{s}\left(h_{s}+0.5 h_{f}\right)}, \quad \lambda_{f}=u_{f} \pm \sqrt{\beta_{f}\left(h_{f}+0.5 h_{s}\right)}
$$

It is important to note that the eigenvalues (24) are exactly the same phase-eigenvalues for solid- and fluidphases as derived by Pudasaini (2012). However, here, these eigenvalues are determined by using much less assumptions than in Pudasaini (2012). Again, note that, in the limit, when the fluid volume fraction vanishes, $\lambda_{s}$ reduces to the classical single-phase granular flow eigenvalues, and in the limit when the solid volume vanishes, $\lambda_{f}$ reduces to the classical single-phase fluid in 24 reduces to the classical single-phase fluid flow eigenvalues. They, respectively, are

$$
\lambda_{s}=u_{s} \pm \sqrt{\beta_{s} h_{s}}, \quad \lambda_{f}=u_{f} \pm \sqrt{\beta_{f} h_{f}} .
$$

It is worth to note that the solid-wave celerity in 24 is enhanced by one-half of the fluid height $h_{f} / 2$ in comparison to the wave celerity of the single phase granular flows in 25); the same holds for the fluidphase wave-celerity. Relevant analysis and discussions on the eigen-structure of two-phase and multi-layer flows can be found in, including, Audusse (2005), Pitman and Le (2005), Bouchut and Morales (2008), Fernandez-Nieto et al. (2008), Pelanti et al. (2008), Castro et al. (2010), and Castro-Diaz et al. (2011).

The fourth set of eigenvalues: The fourth set of eigenvalues for the solid and the fluid phase have three different cases. From (24), the corresponding solid and fluid eigenvalues for Case $\mathbf{A}$ are:

$$
\lambda_{s}=u_{s} \pm \sqrt{0.5 \beta_{s} h_{f}}, \quad \lambda_{f}=u_{f} \pm \sqrt{\beta_{f}\left(h_{f}+0.5 h_{s}\right)} .
$$

There are three interesting aspects in these eigenvalues. First, if the debris flow is largely dominated by the fluid component, the fluid may move faster than the solid (Pudasaini, 2012), as indicated here by the solid- and fluid-phase wave celerities $\sqrt{0.5 \beta_{s} h_{f}}$, and $\sqrt{\beta_{f}\left(h_{f}+0.5 h_{s}\right)}$, respectively. This is reflected by these eigenvalues for situation $\mathbf{A}$. Second, importantly, although the solid lateral pressure gradient is negligible, the fluid lateral pressure (associated with $\beta_{f}$ ) plays crucial role, so does the solid-phase velocity in determining the fluid and the solid-phase eigenvalues. Third, even if the solid pressure gradient could be neglected, there are interactions between the solid and the fluid and thus, even for the solid, the wave celerity is determined by the product of the inter-phase solid and the fluid parameters (variables), $\beta_{s}$ and $h_{f}$.

From (24), the corresponding solid and fluid eigenvalues for Case $\mathbf{B}$ are:

$$
\lambda_{s}=u_{s} \pm \sqrt{\beta_{s}\left(h_{s}+0.5 h_{f}\right)}, \quad \lambda_{f}=u_{f} \pm \sqrt{0.5 \beta_{f} h_{s}} .
$$

The same analysis as in $\mathbf{A}$ can be applied for $\mathbf{B}$; this is the situation, particularly, when the flow (at least locally, say e.g., the solid-dominated front, Pudasaini, 2012) is largely dominated by the solid constituent. This is so, because in this solid-like situation change in the amount of fluid may be negligible. Note that $\mathbf{A}$ is applicable for more fluid-like situation. 


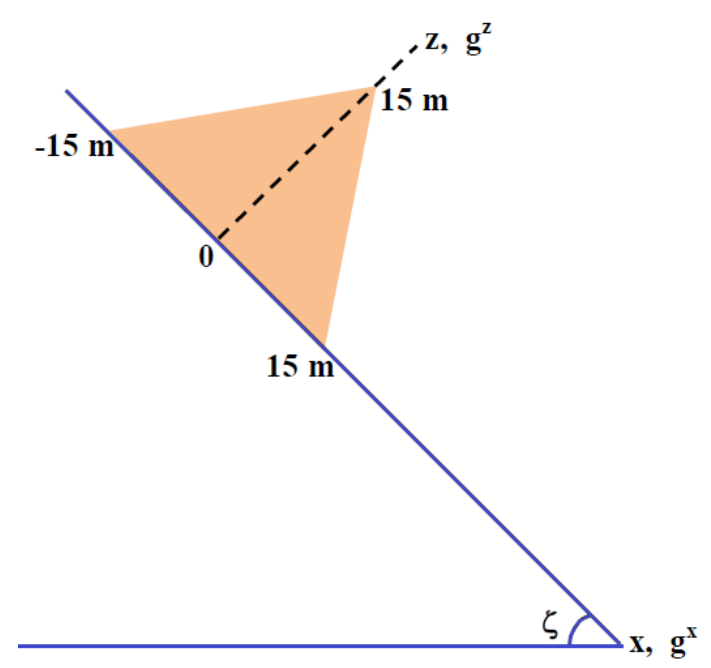

Figure 1: Simulation setup of the two-phase debris flow with initial triangular debris mass consisting of $50 \%$ solid, $50 \%$ fluid, and the inclination of channel is $\zeta=45^{\circ}$.

The situation $\mathbf{C}$ is the produce of $\mathbf{A}$ and $\mathbf{B}$ which is associated with the even more simplified eigenvalues resulting from $(24)$ :

$$
\lambda_{s}=u_{s} \pm \sqrt{0.5 \beta_{s} h_{f}}, \quad \lambda_{f}=u_{f} \pm \sqrt{0.5 \beta_{f} h_{s}}
$$

However, it is important to note that the wave celerities in (28) are determined by the overarching products ( $\beta_{s}$ with $h_{f}, \beta_{f}$ with $\left.h_{s}\right)$ of the inter-phase solid and the fluid parameters (variables). In all these three cases, we observe different types of couplings between the solid and the fluid via the eigenvalues (or the wave celerities). This also strongly implies that the debris motion is characteristically a two-phase flow phenomena. Even more strong couplings are derived in Pokhrel (2014).

\section{Simulation Results}

\subsection{Numerical method, initial set-up and parameter choice}

We implement high-resolution numerical simulation technique based on the widely used total variation diminishing (TVD), shock capturing, non-oscillatory central (NOC) differencing method [27, 34, 36, 40, 42. We consider two different simulation scenarios: (i) with the classical solid-only and fluid-only eigenvalues (15), and (ii) the coupled eigenvalues for solid and fluid phases as given in (14), (16), (24), (26), (27) and (28). The geometrical setting of the simulations is given in Figure 1 initial triangular debris mass is a homogeneous mixture of debris material consisting of $50 \%$ solid and $50 \%$ fluid. For the simulations, the parameters are taken to be

$$
\begin{aligned}
& \delta=15^{\circ}, \quad \phi=35^{\circ}, \quad N_{R}=3 \times 10^{7}, \quad N_{R_{A}}=1.5 \times 10^{7}, \mathcal{C}=0.5, \quad \chi=3, \quad \zeta=45^{\circ}, \\
& \mathcal{P}=0.5, \quad J=1, \quad \rho_{s}=2500 \mathrm{~kg} m^{-3}, \rho_{f}=1100 \mathrm{kgm}^{-3}, \quad R e_{p}=1, \quad U_{T}=1 .
\end{aligned}
$$

The choice of the parameters is based on the physics of debris flows as employed in the Pudasaini (2012), Pudasaini and Krautblatter (2014), Kattel et al. (2016), Kafle et al. (2016) and Mergili et al. (2017).

\subsection{Description of the result}

Considering the geometrical and physical setting as discussed in section $\$ 5.1$, we carry on simulations with different set of eigenvalues (14), (16), (24), 26), (27) and (28) of the model. Simulations with classical uncoupled solid only and fluid only eigenvalues are also presented in grey in each of the simulations results 


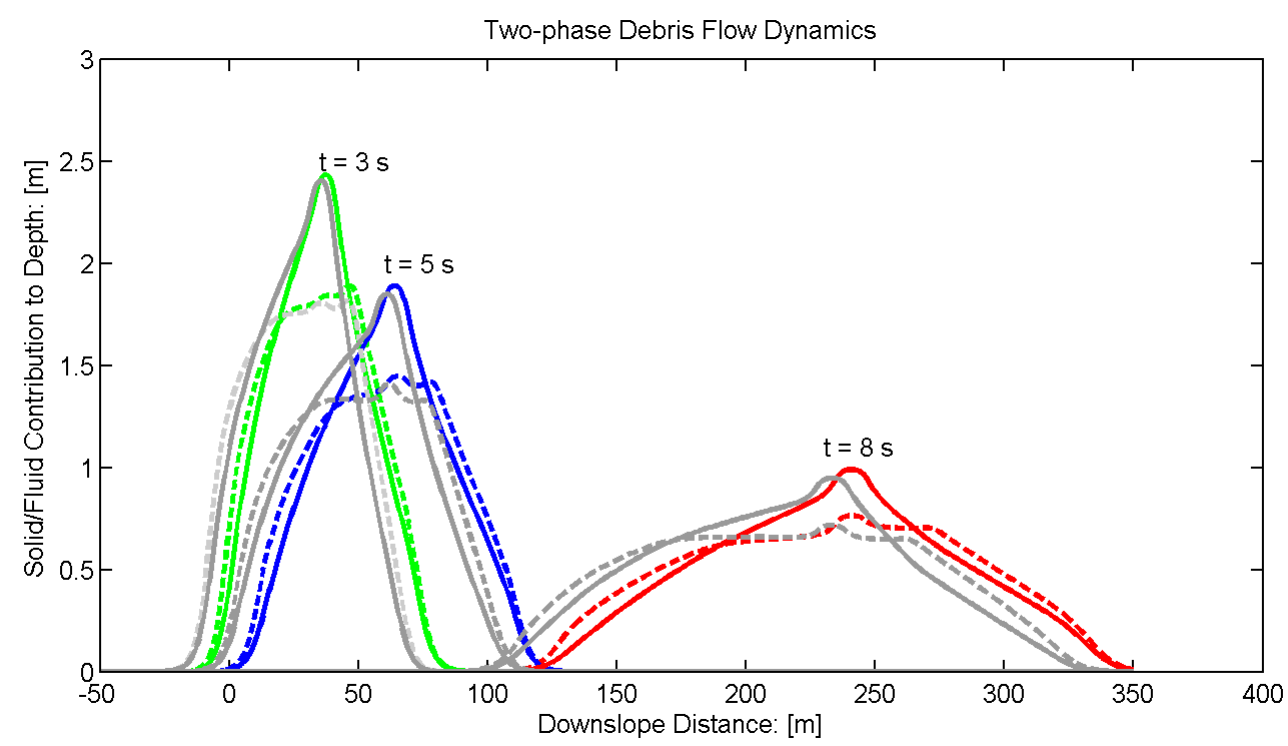

Figure 2: Time and spatial evolution of the solid- and the fluid-phases in a real two-phase debris flow down a channel. The solid and fluid phases are represented by the solid and the dashed lines, respectively. The grey lines are the simulation results as computed with the classical un-coupled solid-only and fluid-only eigenvalues (15). The colour lines are the simulations with the coupled and the enhanced eigenvalues (14) for the solid- and the fluid-phases.

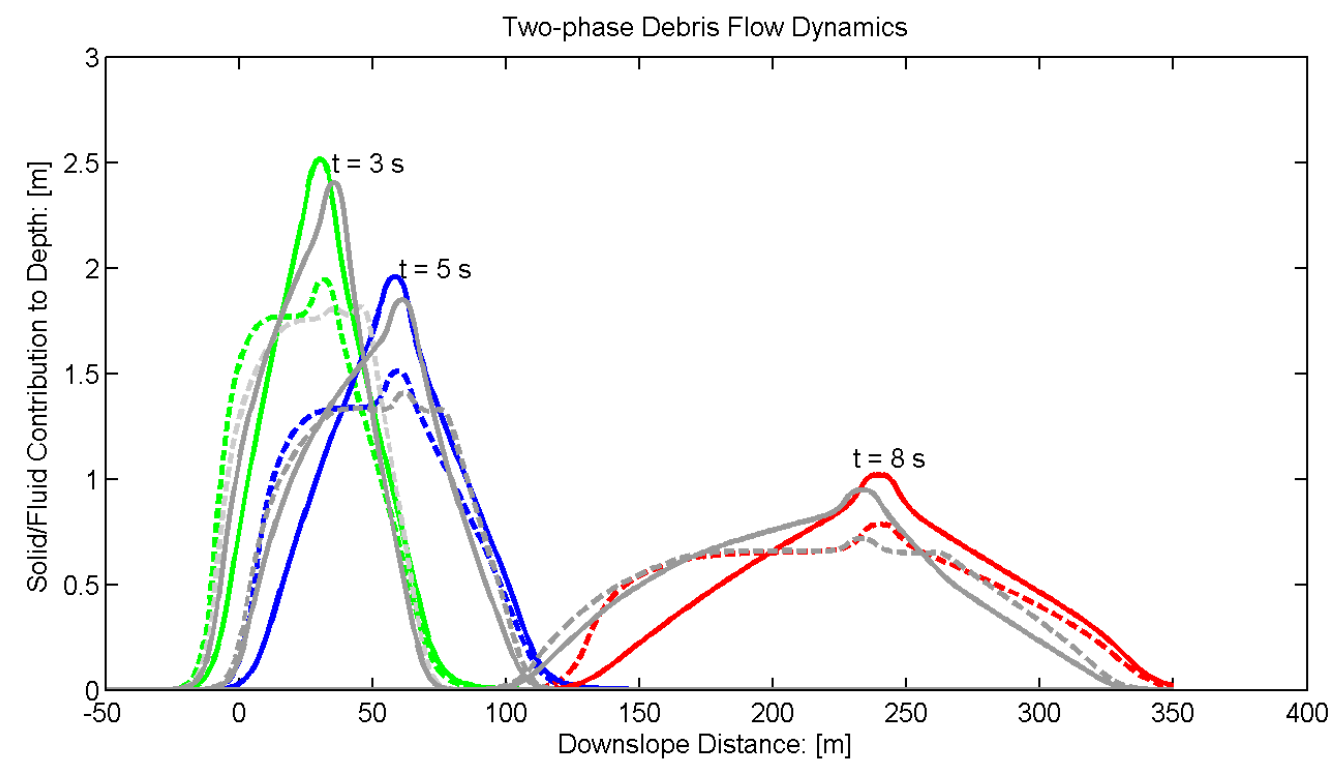

Figure 3: Time and spatial evolution of the solid- and the fluid-phases in a real two-phase debris flow down a channel. The solid and fluid phases are represented by the solid and the dashed lines, respectively. The grey lines are the simulation results as computed with the classical un-coupled solid-only and fluid-only eigenvalues (15). The colour lines are the simulations with the coupled and the enhanced eigenvalues (16) for the solid- and the fluid-phases. 


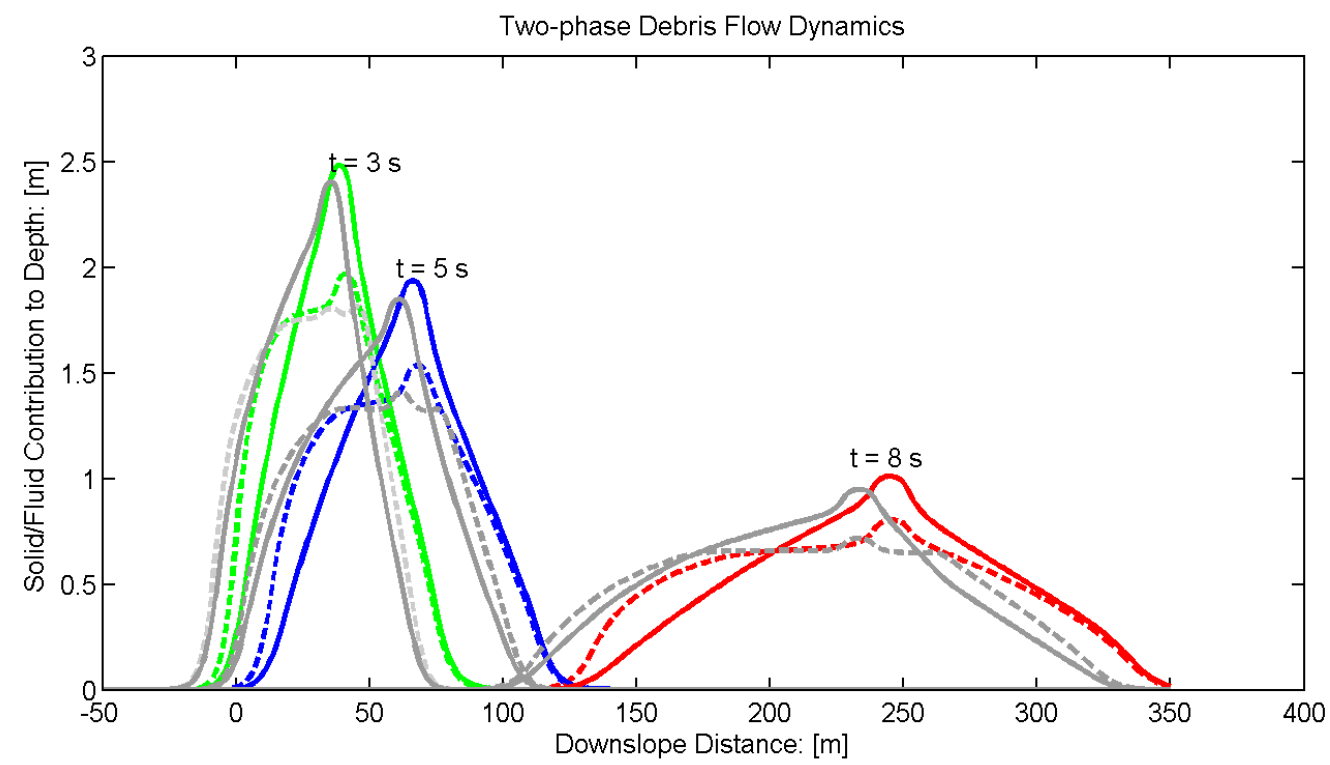

Figure 4: Time and spatial evolution of the solid- and the fluid-phases in a real two-phase debris flow down a channel. The solid and fluid phases are represented by the solid and the dashed lines, respectively. The grey lines are the simulation results as computed with the classical un-coupled solid-only and fluid-only eigenvalues. The colour lines are the simulations with the coupled and the enhanced eigenvalues (24) for the solid- and the fluid-phases.

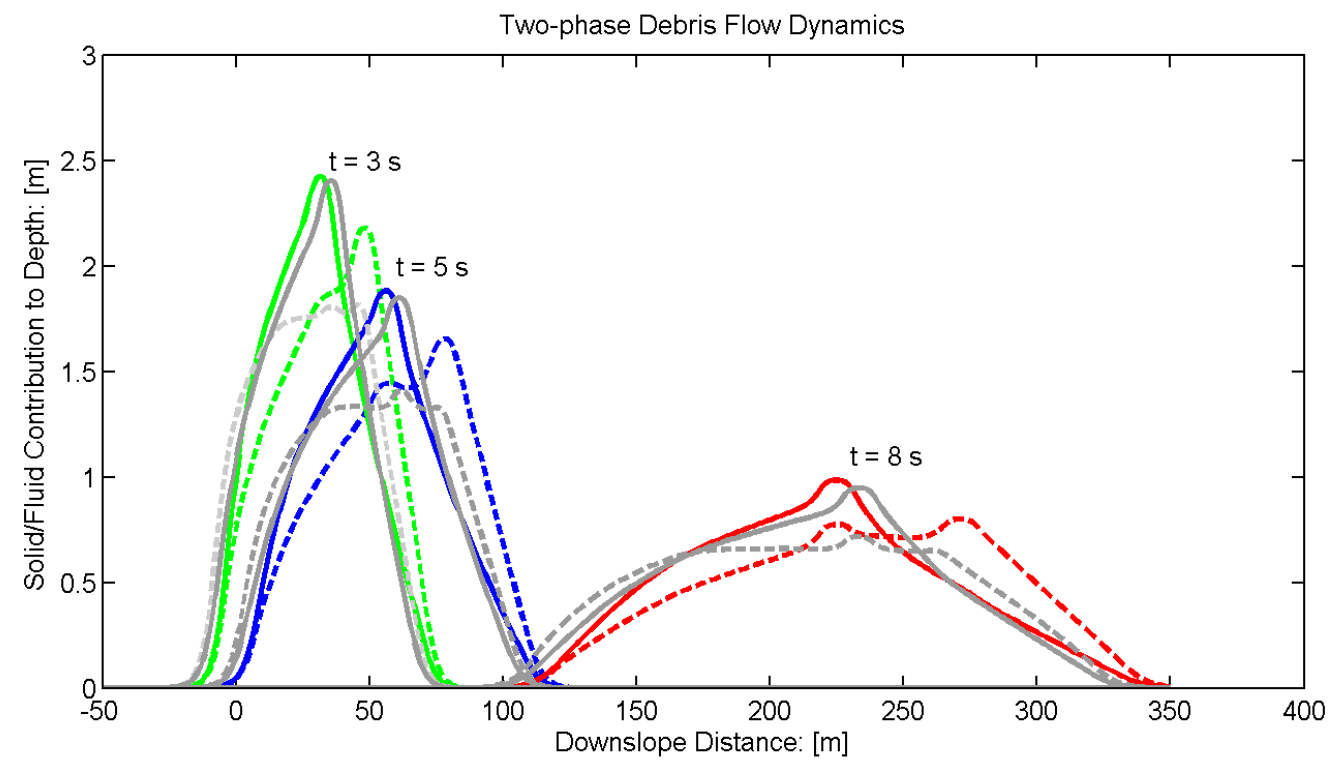

Figure 5: Time and spatial evolution of the solid- and the fluid-phases in a real two-phase debris flow down a channel. The solid and fluid phases are represented by the solid and the dashed lines, respectively. The grey lines are the simulation results as computed with the classical un-coupled solid-only and fluid-only eigenvalues (15). The colour lines are the simulations with the coupled and the enhanced eigenvalues (26) for the solid- and the fluid-phases.

to compare with the simulation results for all the new set of eigenvalues.

Figure 2 displays the simulation results for the two-phase debris flows as it moves down the inclined channel 
Figure 1 using the phase-eigenvalues (14). Here, we analyze in detail the dynamics of the solid and the fluid components in the mixture as shown in Figure 1. After the debris mass is triggered, the solid-phase and the fluid-phase of the debris mixture evolve dynamically differently; the fluid moves faster in the frontand slowly in the backward directions leading to bulging of the fluid in both sides of the debris body. It is observed that the front and tail are dominated by the fluid component whereas the central part of the debris body consists of more solid fraction. Results are shown for the evolution of both the solid- and fluid-phases for $t=3 \mathrm{~s}$ (green), $t=5 \mathrm{~s}$ (blue) and $t=8 \mathrm{~s}$ (red), respectively, in which the solid lines represent the dynamics of the solid phase, and dashed lines represent the dynamics of the fluid phase. The grey lines correspond to the simulations of the two-phase debris flows in which the eigenvalues are considered to be the classical un-coupled solid-only and fluid-only eigenvalues as given in (15). After the debris mass collapse, both solid and fluid spread to the leading and trailing edges of the debris body (both in classical solid only and fluid only (15) and the first set coupled eigenvalues (14). Both the leading and trailing edges are dominated by fluid; fluid shows more dispersion than solid grains have more resistance due to friction (basal and internal); the flow advects downslope direction as time progress and the geometry of the flow has been changing. As a result, the middle part of the debris body losses some fluid and is solid-rich. This solid-rich middle part of the debris body induces drag and hinders the fluid flow of the rear part. Because of this rear part of the debris body is dominated by fluid. In each time slices $(t=3$ $\mathrm{s}, 5 \mathrm{~s}$ or $8 \mathrm{~s}$ ), the peak solid height exceeds the peak fluid height because of the less dispersion of solid mass than fluid. As time progress the leading and the trailing edges of both solid and fluid substantially advects downslope; the increasing distance between the rear and the front part of the debris is due to the dispersion of the solid and fluid masses. This results in the decrease of the peak heights; the solid peak heights decrease from nearly $2.5 \mathrm{~m}(t=3 \mathrm{~s})$ to $1 \mathrm{~m}(t=8 \mathrm{~s})$; the solid surge heads are located at $x=46$ $\mathrm{m}$ at $t=3 \mathrm{~s}, x=71 \mathrm{~m}$ at $t=5 \mathrm{~s}$ and $x=245 \mathrm{~m}$ at $t=8 \mathrm{~s}$. On comparing the results with classical eigenvalues (15), the solid peak heights are nearly same at $t=3 \mathrm{~s}$ and $t=5 \mathrm{~s}$ but a bit less at $t=8 \mathrm{~s}$. This is because, there is larger dispersion of solid mass at $t=8 \mathrm{~s}$ in case of classical eigenvalues (15); the fluid has shown multiple surges around the middle to frontal part of the debris body. The major difference in the results due to the different eigenvalues of the model system is in the difference of the position of the leading and the trailing edges. In each times slice, the position of these edges are a bit farther downslope in case of solid- and fluid- phase coupled eigenvalues (colored plots) than in the case of solid- only and fluidonly classical eigenvalues (grey plots). At $t=8 \mathrm{~s}$, the red (solid and dashed) lines has the leading edges nearly at $x=350 \mathrm{~m}$ whereas the grey lines have those nearly at $x=333 \mathrm{~m}$ only. Thus, the flow is more mobile and travels much faster for both the solid and the fluid phases (because they are coupled) with the coupled and enhanced eigenvalues (14) than with the classical uncoupled solid only and fluid only set of eigenvalues (15. In all of the following simulations results the classical solid- and fluid-phase dynamics using classical solid only and fluid only eigenvalues (15) are also kept to compare with each of the other set of coupled solid-phase and flui-phase eigenvalues $(16),(24),(26), 27)$ and $(28)$.

Figure 3 plots the simulation results for the two-phase debris flows as it moves down the inclined channel Figure 1 using the phase-eigenvalues (16). Results are shown for the evolution of both the solid- and fluidphases for $t=3 \mathrm{~s}$ (green), $t=5 \mathrm{~s}$ (blue) and $t=8 \mathrm{~s}$ (red), respectively, in which the solid lines represent the dynamics of the solid phase, and dashed lines represent the dynamics of the fluid phase. The grey lines correspond to the simulations of the two-phase debris flows in which the eigenvalues are considered to be the classical un-coupled solid-only and fluid-only eigenvalues (15). The front and tails of the solid and fluid are faster downslope at all the time slices as compared to Figure 2 . At $t=3 \mathrm{~s}$ and $5 \mathrm{~s}$, the solid is a bit less dispersed with slight increase of peak heights but the fluid peak heights are substantially increased. It is interesting to observe that at $t=8 \mathrm{~s}$, the solid peak heights are nearly same but the fluid peak height is a bit increased as compared with Figure 2 (with eigenvalues (14)). The solid and fluid flow is even more enhanced in this case as compared with Figure 2 and substantially enhanced as compared to the case of classical eigenvalues (grey lines); the leading edge for both solid fluid reach the downslope distance of $x=350 \mathrm{~m}$.

The simulation results using the phase-eigenvalues (24) are presented in Figure 4. In this case, the leading and trailing edges of the solid and fluid for all time slices are less farther downslope as compared to Figure 3 . the peak heights of both solid and fluid are noticeably decreased at $t=3 \mathrm{~s}$ and $t=5 \mathrm{~s}$. On contrary at $t=8 \mathrm{~s}$, the peak heights are almost same. Although the flow dynamics, here, is a bit enhanced as compared 


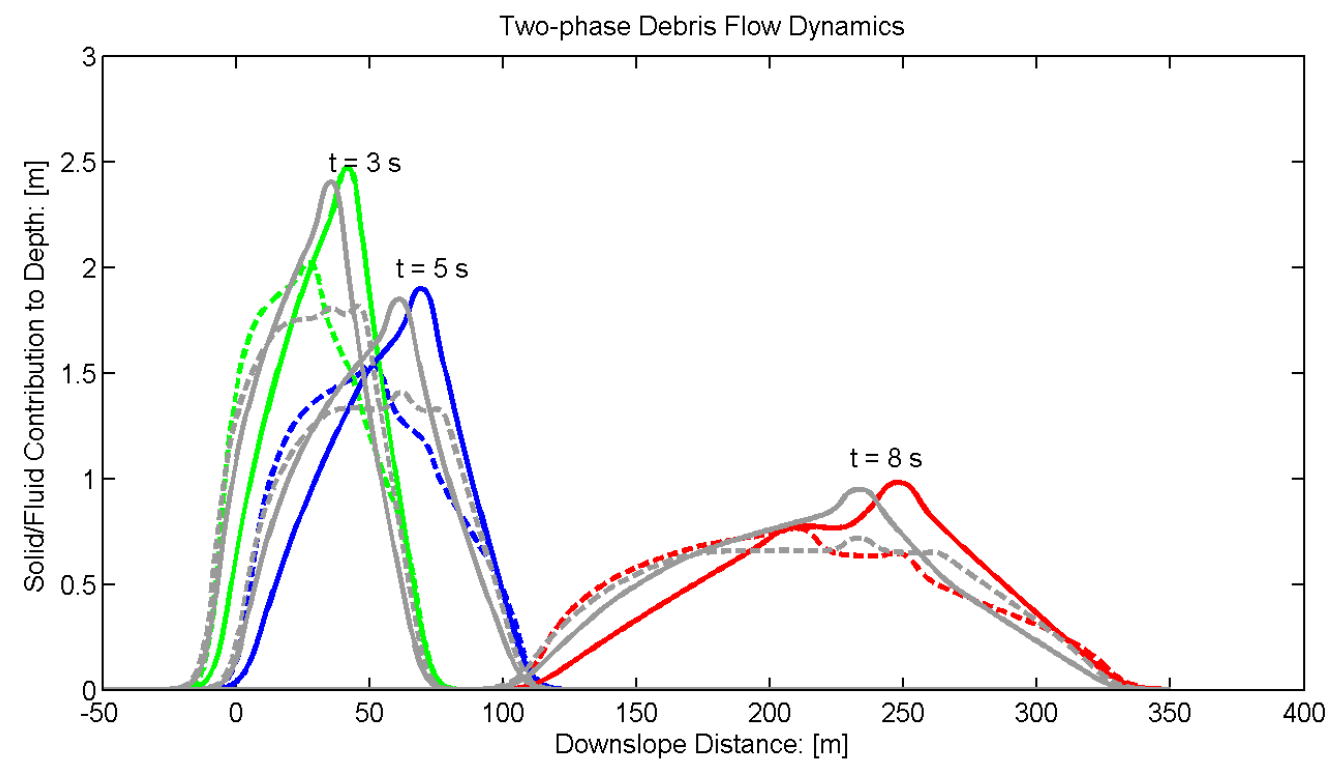

Figure 6: Time and spatial evolution of the solid- and the fluid-phases in a real two-phase debris flow down a channel. The solid and fluid phases are represented by the solid and the dashed lines, respectively. The grey lines are the simulation results as computed with the classical un-coupled solid-only and fluid-only eigenvalues (15). The colour lines are the simulations with the coupled and the enhanced eigenvalues (27) for the solid- and the fluid-phases.

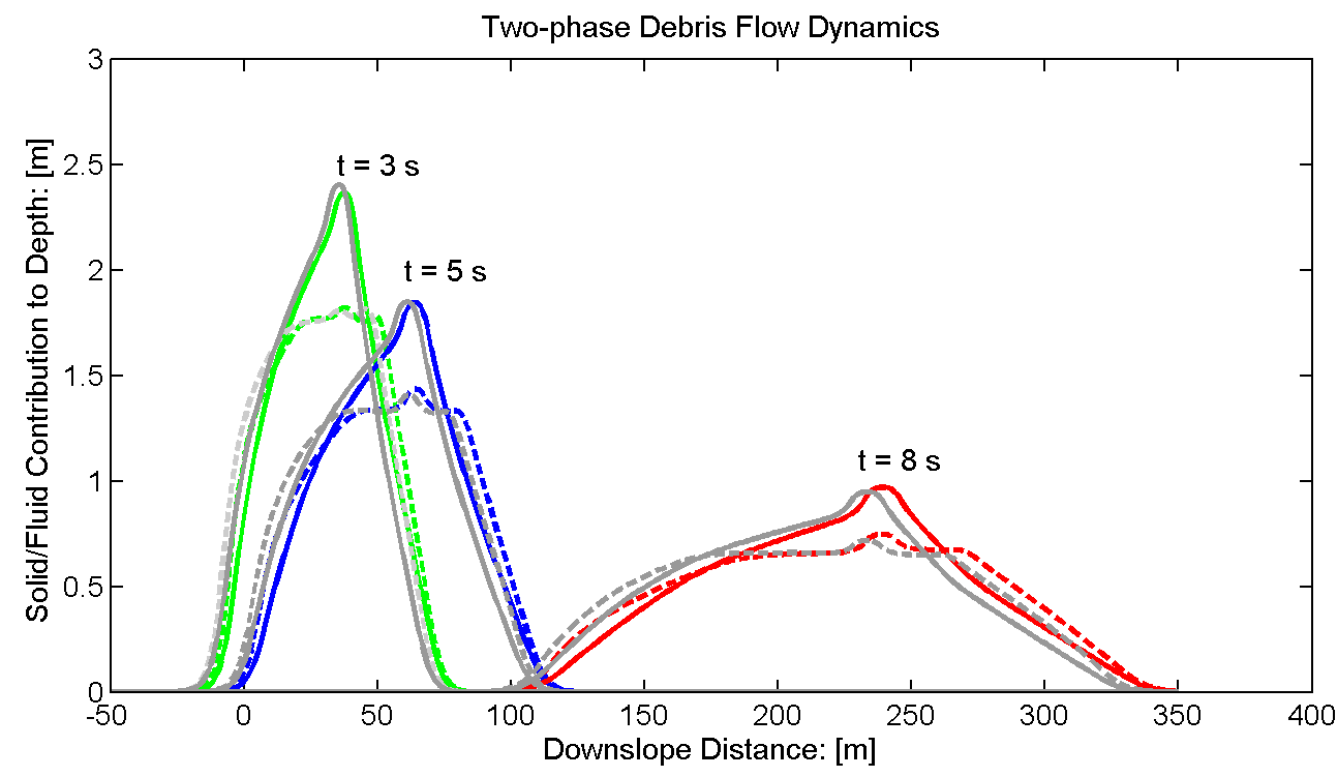

Figure 7: Time and spatial evolution of the solid- and the fluid-phases in a real two-phase debris flow down a channel. The solid and fluid phases are represented by the solid and the dashed lines, respectively. The grey lines are the simulation results as computed with the classical un-coupled solid-only and fluid-only eigenvalues (15). The colour lines are the simulations with the coupled and the enhanced eigenvalues (28) for the solid- and the fluid-phases.

to the classical eigenvalues (15), but it is less enhanced as compared to the eigenvalues (16).

Figures (5) - (7) present the results by using the sets of eigenvalues in case (A) 26), case (B) 27) and case (C) 
(28) respectively. The solid- and fluid-phase dynamics remain almost same in these figures. Although the flow is little bit enhanced as compared to the classical eigenvalues, but are less enhanced than the second set of eigenvalues 16p (Figure 3). However, a close scrutinizing of the leading edges of the solid- and fluidphases in $t=8 \mathrm{~s}$ shows that, the fluid phase is little more enhanced in Figure 5 and the solid-phase is little more enhanced in Figure 6 as compared with Figure 5 and Figure 6 and also with Figure 7

These simulation results show that the solid- and fluid-phase coupled eigenvalues $(14),(16),(24),(26)$, (27) and 28) provide enhanced flow dynamics as compared to the classical solid only and fluid only eigenvalues (15). Among them the second set of eigenvalues (16) (Figure 3) gives the most enhanced flow dynamics. As the eigenvalues determine the wave speeds of the flow which eventually determine the intrinsic simulation dynamics of the flow via the CFL conditions, with the physically more appropriate coupled eigenvalues, in which the solid and the fluid eigenvalues are coupled to each-other via, e.g., the geometric evolutions of the solid and the fluid components and the other physical parameters, the flow mobility is substantially enhanced as measured, e.g., from the frontal distances of the evolution of the solid and the fluid phases in both simulation scenarios. This, then clearly indicates that the physically more meaningful and complete eigenvalues are required for the reliable prediction of the fluid dynamical variables, to appropriately determine the enhanced flow dynamical quantities, including the evolution of the solidand fluid-phase, fluid volume fraction, total debris and landslide height, inundation area, phase velocities, and the impact forces. A formally obtained eigenvalues of a system of partial differential equations (PDEs) contain two different contributions: kinetic energy (thus the relevant velocity of solid-fluid) and the potential energy (i.e., pressure potential energy so the wave speed ). For a single phase flows such as shallow water flows or granular flows or landslides, avalanche and single-phase debris flows, the wave celerity ( for long wave ) is simply given by the square root of the product of the pressure factor $\beta=\rho g \cos \zeta K$ and the flow depth $h$, i.e., $\sqrt{\beta h}$. However, for the coupled mixture flows it is not clear what is the wave celerity. The most legitimate way of obtaining the wave celerity of a system is to compute the eigenvalues of the system of PDEs representing the mixture flows. More general eigenvalues of the real two-phase model can be found in Pokhrel (2014).

\section{Conclusions}

We have derived several coupled solid- and fluid-phase eigenvalues for the real two-phase debris flow model by using rational factorization method in different assumptions, and also presented some analytically derived coupled solid- and fluid-phase eigenvalues for the real two-phase debris flow model [35. Simulation results using these phase-eigenvalues influenced substantially in the dynamical behavior of the debris mass flows. The correct and enhanced descriptions of the mass flows by these eigenvalues were derived by including the solid and the fluid properties of the mixture. This is one of the main aspects of this contribution. High-resolution, shock-capturing scheme has been implemented to solve the model equations numerically. Advanced computational results has been presented and compared with the new coupled phase-eigenvalues for the solid and fluid-phases, and the pre-existing un-coupled solid-only and fluid-only eigenvalues. The results clearly indicates that the coupled eigenvalues are necessary to appropriately simulate real two-phase debris flows, to enhanced determination the flow mobility and inundation areas.

Acknowledgments: Puskar R. Pokhrel acknowledges University Grant Commission (UGC), Nepal for the financial support provided as a PhD fellowship (PhD - 2071/072 - Sci. \& Tech. - 01). We gratefully acknowledge Mr. Parameshwari Kattel for his valuable suggestions in this work.

\section{References}

[1] Audusse, E., 2005, A multilayer Saint-Venant model: Derivation and numerical validation. Discrete Cont. Dyn. Syst., Ser., B5(2), 189-214.

[2] Bouchut, F. and Morales, T., 2008, An entropy satisfying scheme for two layer shallow water equations with uncoupled treatment. ESAIM: Math. Modell. Numer. Anal., 42(4), 683-698. 
[3] Castro, M., Frings, J.T., Noelle, S., Pares, C. and Puppo, G., 2010, On the hyperbolicity of two- and three-layer shallow water equations. Inst. fr Geom. und Prakt. Math. Aachen Univ., Rep.314:8.

[4] Castro-Diaz, M.J., Fernandez-Nietom, E.D., Gonzalez-Vida, J.M. and Pares-Madronal, C., 2011, Numerical treatment of the loss of hyperbolicity of the two-layer shallow-water system. J. Sci. Comput., 48(1-3), 16-40.

[5] Crosta, G.B., Chen, H. and Lee, C.F., 2004, Replay of the 1987 Val Pola Landslide. Italian Alps. Geomorphology, 60(12), 127-146.

[6] Crosta, G.B., Imposimato, S. and Roddeman, D., 2003, Numerical modelling of large landslides stability and runout. Nat.Hazards Earth Syst. Sci., 3, 523-538.

[7] Domnik, B., Pudasaini, S.P., Katzenbach, R. and Miller, S.A., 2013, Coupling of full two-dimensional and depth-averaged models for granular flows J. Non-Newtonian Fluid Mechanics, 201, 56-68.

[8] Domnik, B. and Pudasaini, S.P., 2012, Full two-dimensional rapid chute flows of simple viscoplastic granular materials with a pressure-dependent dynamic slip-velocity and their numerical simulations. $J$. Non-Newtonian Fluid Mech., 173-174, 72-86.

[9] Fernandez-Nieto, E.D., Bouchut, F., Bresch, D., Castro Daz, M.J. and Mangeney, A., 2008, A new Savage-Hutter type model for submarine avalanches and generated tsunami. J. Comput. Phys., 227(16), 7720-7754.

[10] Govi, M.G. and Nicoletti, P.G., 2002, Val Pola rock avalanche of July 28, 1987, in Valtellina (Central Italian Alps). Geological Society of America, Review in Engineering Geology, 15, 71-90.

[11] Hungr, O., McDougall, S. and Bovis, M., 2005, Entrainment of material by debris Flows. In Debrisflows Hazard and related Phenomena, ed. M. Jakob and O. Hungr. Praxis, Springer, Berlin.

[12] Hutter, K. and Schneider, L., 2010 a, Important Aspects in the Formulation of Solid-Fluid Debris-Flow models, Part I: Thermodynamic Implications. Continuum Mech. Therm., 22, 363-390.

[13] Hutter, K. and Schneider, L., 2010 b, Important Aspects in the Formulation of Solid-Fluid Debris-Flow models, Part II: Constitutive Modeling. Continuum Mech. Therm., 22, 391-411.

[14] Hutter, K., Svendsen, B. and Rickenmann, D., 1996, "Debris flow modelling: A review". Continuum Mech. Thermodyn., 8, 1-35.

[15] Iverson, R.M., 2012, Elementary theory of bed-sediment entrainment by debris flows and avalanches. J. Geophys. Res., 117 (F03006), 197-200.

[16] Iverson, R.M. and Denlinger, R.P., 2001, Flow of variably fluidized granular masses across threedimensional terrain: 1. Coulomb mixture theory. J. Geophys. Res., 106(B1), 537-552.

[17] Johnson, A.M., 1970, Physical Processes in Geology Freeman \& Cooper, San Francisco, California, United States of America.

[18] Kafle, J., Pokhrel, P.R., Khattri, K.B., Kattel, P., Tuladhar, B.M. and Pudasaini, S.P., 2016, Landslidegenerated tsunami and particle transport in mountain lakes and reservoirs. Ann. Glaciol, 57, $232-244$.

[19] Kattel, P., Khattri, K.B., Pokhrel, P.R., Kafle, J., Tuladhar, B.M. and Pudasaini, S.P., 2016, Simulating glacial lake outburst floods with a two-phase mass flow model. Ann. Glaciol., 57, 349-358

[20] Kowalski, J. and McElwaine, J.N., 2013, Shallow two-component gravity-driven flows with vertical variation. J. Fluid Mech., 714, 434-462.

[21] Kowalski, J., 2008, Two-phase Modeling of Debris Flows. PhD Dissertation, University of Augsburg, Augsburg. 
Determination of Phase-Eigenvalues by Rational Factorization and Enhanced Simulation of Two-Phase Mass Flow

[22] McCoy, S.W., Kean, J.W., Coe, J.A., Tucker, G.E., Staley, D.M. and Wasklewicz, T.A., 2012, Sediment entrainment by debris flows: In situ measurements from the headwaters of a steep catchment. J. Geophys. Res., 117(F03016).

[23] Mergili, M., Fischer, J.-T., Krenn, J. and Pudasaini, S.P., 2017, r.avaflow v1, an advanced open-source computational framework for the propagation and interaction of two-phase mass flow. Geosci. Model Dev., 10, 553-569.

[24] Mergili, M., Schratz, K., Ostermann, A. and Fellin, W., 2012, Physically-based modeling of granular flows with Open Source GIS. Nat. Hazards Earth Syst. Sci., 12, 187-200.

[25] Miller, D.J., 1960, Alaska Earthquake on July 10, 1958: Giant wave in Lituya Bay. Bull. Seismol. Soc. Am., 50(2), 253-266.

[26] Miller, D.J., 1964, Alaska Earthquake on July 10, 1958: Giant wave in Lituya Bay. Bull. Seismol. Soc. Am., 50(2), 253-266.

[27] Nessyahu, H. and Tadmor, E., 1990, Non-oscillatory central differencing for hyperbolic conservation laws. J. Comput. Phys., 87, 408-463.

[28] O'Brien, J. S., Julien,P.J. and Fullerton, W.T., 1993, Two-dimensional water flood and mudflow simulation, J. Hyd. Eng., 119(2), 244-261.

[29] Pelanti, M., Bouchut, F. and Mangeney, A., 2008, A Roe-Type scheme for two-phase shallow granular flows over variable topography. Math. Model. Numer. Anal., 42, 851-885.

[30] Pitman, E.B. and Le, L., 2005, A two-fluid model for avalanche and debris flows. Philos. Trans. R. Soc. A., 363, 1573-1602.

[31] Pokhrel, P.R., 2014, General eigenvalues and wave speeds for two-phase mass flows: Supercritical and subcritical states. M Phil. Thesis, School of Science, Kathmandu University, Kavre, Nepal.

[32] Pokhrel, P.R., Khattri, K.B., Tuladhar, B.M. and Pudasaini, S.P., 2018, A generalized quasi two-phase bulk mixture model for mass flow. Int. J. of Non-Linear Mech., 99, 229-239.

[33] Pokhrel, P.R. and Pudasaini, S.P., 2019, A simple stream function vorticity - formulation of mixture mass flow. Int. J. of Non-Linear Mech., doi: 10.1016/j.ijnonlinmec.2019.103317.

[34] Pudasaini, S.P., 2011, Some exact solutions for debris and avalanche flows. Phys. Fluids, $23(4), 043301$.

[35] Pudasaini, S.P., 2012, A general two-phase debris flow model, J. of Geophysics Res., 117, F03010.

[36] Pudasaini, S.P. and Hutter, K., 2007, Avalanche Dynamics: Dynamics of Rapid Flows of Dense Granular Avalanches, Springer-Verlag, Berlin.

[37] Pudasaini, S.P. and Miller, S.A., 2012a, Buoyancy Induced Mobility in Two-phase Debris Flow. American Institute of Physics Proceedings, 1479, 149-152.

[38] Pudasaini, S.P. and Miller, S.A., 2012b, A real two-phase submarine debris flow and tsunami. American Institute of Physics Proceedings, 1479, 197-200.

[39] Pudasaini, S.P. and Miller, S.A., 2013, The hypermobility of huge landslides and avalanches. Engineering Geology, 157, 124-132.

[40] Pudasaini, S.P., Wang, Y. and Hutter, K., 2005, Modeling debris flows down general channels. Nat. Hazards Earth Syst. Sci., 5, 799-819.

[41] Reid, M.E., Iverson, R.M., Logan, M., Lahusen, R.G., Godt, J.W. and Griswold, J.P., 2011, Entrainment of bed sediment by debris flows: results from large-scale experiments. Italian J. Eng. Geol. Env., doi:10.4408/IJEGE.2011-03.B-042. 
[42] Tai, Y.-C., Noelle, S., Gray, J.M.N.T. and Hutter, K., 2002, Shock capturing and front-tracking methods for granular avalanches. J. Comput. Phys., 175, 269-301.

[43] Takahashi, T., 1981, Debris flow: Annual Review, Fluid Mechanics, 13, 57-77.

[44] Takahashi, T., 2007, Debris Flow: Mechanics, Prediction and Countermeasures. Taylor and Francis, New York.

[45] Wang, Y., Pudasaini, S.P., Hsiau, S.S. and Hutter, K., 2005, Velocity measurements in dry granular avalanches usingparticle image velocimetry technique and comparison with theoretical redictions. Physics of Fluid 27, no. 1-2: 301-313.

[46] Weiss, R., Fritz, H.M. and Wuennemann, K., 2009, Hybrid modeling of the mega-tsunami runup in Lituya Bay after half a century. Geophys. Res. Lett., 36, L09602. 